Revista de Psicología Vol. 36 (2), 2018 (ISSN 0254-9247)

\title{
Orientación temporal y metas vitales en estudiantes argentinos ${ }^{1}$
}

\author{
Hilda Difabio de Anglat ${ }^{2}$, Stella Maris Vázquez ${ }^{3}$ Marianela Noriega Biggio ${ }^{4}$ \\ Centro de Investigaciones Cuyo ${ }^{2}$, Centro de Investigaciones en Antropología \\ Filosófica y Cultural (CIAFIC) ${ }^{3,4}$, CONICET $T^{2,3}$
}

El presente estudio tuvo como objetivos verificar la relación entre orientación temporal y calidad de metas vitales y el rol de la edad, el sexo y el nivel de planificación; así como la influencia de la calidad de las metas vitales y el sexo sobre la extensión temporal. Se aplicó el Inventario de Perspectiva Temporal de Zimbardo \& Boyd, algunos ítems del Inventario de Perspectiva Temporal Futura Trascendental de Boyd \& Zimbardo y el Cuestionario de metas y temores de Nurmi. Mediante análisis de conglomerados y de varianza, se encontró que hay un estilo de orientación temporal más adaptativo, que influye positivamente sobre la planificación y que las variaciones de mayor relevancia aparecen cuando se considera el peso de la cualidad de las metas sobre la orientación temporal en interacción con la edad y el sexo. Palabras clave: orientación temporal, metas vitales, diferencias de género, diferencias etáreas.

\section{Temporal orientation and life goals for Argentine students}

The present study aims to verify the relationship between temporal orientation and quality of vital goals and the role of age, sex and planning level. Also, it aims to study the influence of the vital goals' quality and the sex on the temporal extension. The Zimbardo \& Boyd Time Perspective Inventory was applied, as well as some items from the Boyd \& Zimbardo Transcendental Future Time Perspective Inventory and the Nurmi Hopes and fears

1 Esta investigación fue financiada por CONICET con fondos provenientes del Proyecto de Investigación $11220130100503 \mathrm{CO}$ Motivación académica en relación con perspectiva futura y aprendizaje autorregulado en estudiantes universitarios.

2 Doctora en Ciencias de la educación. Directora del Centro de Investigaciones Cuyo. Dirección Postal: Primitivo de la Reta $5222^{\circ} \mathrm{K}$ (Código postal: 5.500) Mendoza, Argentina. Contacto: centroinvestigacionescuyo@gmail.com; ganglat@gmail.com.

3 Doctora en Filosofía. Directora del Departamento de Filosofía de la Educación del Centro de Investigaciones en Antropología Filosófica y Cultural (CIAFIC). Dirección postal: Lacroze 2100, (1426) Ciudad Autónoma de Buenos Aires, Argentina. Contacto: stellavazquez@ gmail.com.

4 Magíster en Tecnología y Metodología de la formación en red por la Universidad Ca’Foscari. Personal de apoyo del Centro de Investigaciones en Antropología Filosófica y Cultural (CIAFIC). Dirección postal: Federico Lacroze 2100, (1426) Ciudad Autónoma de Buenos Aires, Argentina. Contacto: marianelanoriega@gmail.com. 
Questionnaire. By means of cluster and variance analysis, it was found that there is a more adaptive temporal orientation style, which influences planning and that the most relevant variations appear when considering the weight of the goals' quality on time orientation in interaction with age and sex.

Keywords: temporal orientation, vital goals, gender differences, age differences.

\section{Orientaçáo temporal e metas de vida para estudantes argentinos}

O presente estudo teve como objetivos verificar a relaçáo entre a orientação temporal e a qualidade das metas vitais e o papel da idade, sexo e nível de planejamento; bem como a influência da qualidade dos objetivos vitais e do sexo na extensão temporal. O Inventário da Perspectiva Temporal de Zimbardo \& Boyd foi aplicado, bem como alguns items do Inventario da Perspectiva Temporal Futura Transcendental de Boyd \& Zimbardo e do Questionário de metas e medos de Nurmi. Por meio da análise de cluster e variância, verifica-se que existe um estilo da orientaçáo temporal mais adaptativo, que influencia positivamente o planejamento e que as variaçóes mais relevantes aparecem quando se considera o peso da qualidade das metas na orientação temporal em interação com idade e sexo.

Palavras-chave: orientação temporal, objetivos vitais, diferenças de gênero, diferenças de idade.

\section{Orientation temporelle et objectifs de vie pour les étudiants argentins}

La présente étude avait pour objectifs étaient de vérifier la relation entre l'orientation temporelle et la qualité des objectifs de vie et le rôle de l'âge, du sexe et du niveau de planification; ainsi que l'influence de la qualité des buts de vie et du sexe sur l'extension temporelle. L'inventaire de perspective temporelle de Zimbardo et Boyd a été appliqué, ainsi que certains items de l'Inventaire de perspective temporelle transcendantale de Boyd \& Zimbardo et du Questionnaire Espoirs et craintes, de Nurmi. Au moyen d'une analyse de clusters et de variance, on constate qu'il existe un style d'orientation temporelle plus adaptatif, qui influence positivement la planification et que les variations les plus pertinentes apparaissent lorsqu'on considère le poids de la qualité des objectifs sur la orientation temporelle, en interaction avec l'âge et le sexe.

Mots-clés: orientation temporelle, objectifs vitaux, différences entre les sexes, différences d'âge. 
La dimensión temporal es abordada por diversas ciencias, desde la física a la filosofía o la psicología. En cualquiera de ellas, el tiempo refiere siempre al cambio, al devenir y una definición clásica, a la que remiten todos los ámbitos de su estudio, es justamente la de Aristóteles (1995), en cuyo concepto el tiempo es la medida del cambio, que es advertido porque hay algo que permanece. El presente estudio se centra en la perspectiva psicológica, es decir, del tiempo en cuanto humano, el cual no puede prescindir del anclaje en lo que es común a todo concepto de tiempo, ya que el tiempo humano supone la persona, tanto en sentido óntico, substancial, cuanto en la vivencia, más o menos clara, de la propia identidad, vale decir la percepción de que el presente es continuidad desde el pasado que se hace presente en el ahora y aquí y hacia el futuro que es presente virtual.

Así como el tiempo físico es la medida del cambio en las cosas materiales, el tiempo humano es la medida, vivenciada, del devenir de la vida, de su duración en cuanto captada en unidad (Komar, 2003), por lo cual, en la experiencia humana, el tiempo nunca es un puro fluir. Dicho de otro modo, solo en el hombre el tiempo se hace realidad conciente y adquiere una suerte de permanencia, derivada de la advertencia de que algo cambia a partir de la realidad permanente de la persona.

La dimensión del tiempo, que es esencial en el desarrollo de la vida psíquica, espiritual y social del hombre, fue dejada en la sombra en el ámbito de la psicología, sobre todo en las décadas en que predominaron los enfoques conductistas -centrados en el presente fáctico- y psicoanalíticos-centrados en el pasado- y cobra relevancia a partir de la psicología fenomenológica humanista y cognitivista.

\section{La dimensión temporal}

Los autores se refieren al tema con distintas denominaciones, que implican enfoques teóricos diversos o matices que buscan subrayar 
distintos aspectos. Lewin (1935) llama perspectiva temporal a la totalidad de los criterios del individuo acerca de su futuro y pasado psicológicos existentes en un momento dado y señala que es uno de los aspectos fundamentales del desarrollo. A medida que se producen cambios en la estructura cognitiva, es posible proyectarse hacia un futuro cada vez más distante. Así, el niño pequeño vive casi exclusivamente en el presente, incluyendo el pasado y futuro inmediatos, con una limitación que Lewin considera propia de la conducta primitiva.

Por otro lado, Fraisse (1983) usa la denominación horizonte temporal, distinguiendo el presente vivido y el percibido, así como el futuro inmediato y el prospectivo, que es vivido en forma de representación. Designa como futuro prospectivo el que corresponde a objetivos que no son alcanzables en el presente y que constituyen lo que denominamos sus perspectivas temporales, o mejor, su horizonte temporal (Fraisse, 1989). Ambos autores miran en particular la función cognitiva ligada a la dimensión temporal de la conducta. Nuttin (1980), por una parte, introduce importantes distinciones terminológicas y, por otra, en su posición teórica integra la función afectivo-tendencial a la cognitiva, a partir de fundamentos antropológicos. Puntualiza:

Algunos autores tienden a reunir en una sola voz -"perspectiva"- tres aspectos diferentes del tiempo psicológico. Nosotros, contrariamente, proponemos distinguir entre la perspectiva como tal (con sus atributos esenciales como la profundidad o extensión, la densidad, la estructura y el grado de realidad), por un lado, y, por el otro, la actitud temporal y la orientación predominante hacia el pasado, el presente o el porvenir (Nuttin, 1980, p. 5).

Esta distinción parece pertinente, ya que el término perspectiva refiere, en primer lugar, al espacio y luego, por analogía, al tiempo. En ambos casos la perspectiva menciona un modo de ver algo en forma panorámica, sobre todo cuando está distante.

La perspectiva temporal, según Nuttin (1980), consiste en la percepción como presente de lo que se da en una sucesión y se construye a partir de una representación cognitiva que depende de la elaboración 
de las necesidades y de la formación consecuente de propósitos y proyectos. Vale decir, para satisfacer, por ejemplo, la necesidad de comer, es preciso que esta llegue al plano de la conciencia y, a partir de allí, el sujeto genere un propósito y plan para satisfacerla, desde el nivel más elemental hasta la realización de actividades laborales, económicas, etc. La extensión, como dimensión esencial de la perspectiva temporal, se gesta a partir de las distancias temporales de esos objetos-meta —en qué lugar del tiempo futuro cada sujeto ubica la meta- . Es decir que lo decisivo es el contenido de la meta: una meta como la de realizarse profesionalmente estará, por su naturaleza, más lejana en el tiempo que otra meta que puede referirse, por ejemplo, a reunirse con amigos.

La meta sustenta, da realidad a la perspectiva temporal y, a la vez, permite construir el sentido de identidad y, de modo retrospectivo, integrar en unidad los momentos vividos. De modo recíproco, a medida que dicha perspectiva se extiende, da lugar a la posibilidad de concebir y sostener metas que se ubican en un tiempo no inmediato. Esto permite hablar de una relación dialógica — de retroalimentación- entre las metas vitales y la perspectiva temporal. La presencia virtual de estos objetos-meta a distintas distancias es lo que constituye la perspectiva temporal, al crear en la persona una apertura habitual sobre el futuro o sobre el pasado; en cambio, su ausencia la encierra en un presente continuo.

El presente estudio se centra en la orientación temporal, que autores como Zimbardo y Boyd (1999), identifican con la perspectiva temporal. La orientación temporal, considerada como una dimensión estable, refiere a una preferencia relativa por el presente, pasado o futuro en lo cognitivo, actitudinal y de conducta (Webster, 2011). Ha sido definida como el predominio con que el pasado, presente o futuro ocupan el espíritu del individuo (Thiebaut, 1998), "la dirección preferencial de los pensamientos de un sujeto hacia el presente, pasado o futuro, que ejerce una influencia dinámica sobre su experiencia, motivación, pensamiento, y diversos aspectos de su conducta" (Boniwell, 2009 , p. 295). Debe hacerse notar que el peso relativo que tengan los marcos temporales de pasado o de futuro introduce diferencias con- 
siderables en las decisiones y conductas actuales, por lo que resulta importante explorar las modalidades que adquieren estas dimensiones de la perspectiva $-\mathrm{u}$ orientación - temporal en diferentes personas.

Zimbardo es el autor que más se ha ocupado de la operacionalización de este concepto, que define como "un proceso no consciente mediante el cual el flujo de las experiencias personales y sociales se encuadra en categorías temporales que ayudan a dar orden, coherencia y significado a esas experiencias" (Zimbardo \& Boyd, 1999, p. 1271). Sin embargo, la teoría que sustenta el instrumento de Zimbardo, prescinde del peso que pueda tener en esas orientaciones temporales la naturaleza de las metas. Por otra parte, este enfoque es calificado por algunos autores (André, van Vianen, Peetsma et al., 2018) como general, pues no distingue dominios (por ejemplo, los diferentes dominios en la vida de una persona: trabajo, estudio, salud, relaciones sociales, etc.), o como atemática (Seginer \& Lens, 2015). Se ha considerado que esta preferencia por alguna de las dimensiones puede funcionar como un rasgo estático de personalidad (Zimbardo, Keough \& Boyd, 1997) que implica determinados costos, es decir, que puede llegar a ser no adaptativa (Boniwell, 2009); de allí que los autores introduzcan el concepto de perspectiva temporal balanceada (BTP) (Boniwell \& Zimbardo, 2004; Boyd \& Zimbardo, 2005), según la cual el presente, pasado y futuro se combinan de modo flexible, respondiendo a las exigencias de los momentos y tareas en la vida de las personas, así como a sus valores dominantes (Zimbardo, 2002). Así, por ejemplo, cuando se está planificando una tarea, es de esperar que el sujeto pueda ubicarse con facilidad en el futuro; pero, si se trata de compartir un evento familiar, lo adecuado es situarse en el presente, para disfrutar del momento.

En definitiva, como señalan Zimbardo y Boyd (1999):

El foco en el futuro da alas para remontarse hacia nuevas alturas en los logros, el foco en el pasado positivo establece las raíces con la tradición y fundamenta el sentido de la identidad personal, el foco en el presente hedonista nutre la vida diaria con la alegría de la juventud y los gozos de la sensualidad. Las personas necesitan que todo esto opere de modo armonioso para realizar plenamente su potencial humano (Zimbardo \& Boyd, 1999, p. 1285). 


\section{Metas vitales}

El estilo de orientación temporal, y en particular su extensión, se genera a partir del tipo de metas que las personas se proponen. Las metas se describen (Elliot, Sheldon \& Church, 1997; Wrosch \& Scheier, 2003) como objetivos propios, articulados conscientemente, que dan a la persona un sentido de propósito, estructura e identidad (Chen Zhang \& Yu, 2014), al permitir la dirección del desarrollo de las distintas etapas de la vida (Shulman \& Nurmi, 2010). Así, las metas operan como ejes de organización psicológico-existencial en la medida en que contribuyen a dar significado a la experiencia vivida (Klinger, 1998), lo que depende de modo fundamental de la cualidad de las metas (Vansteenkiste, Simons, Lens et al., 2004; Lens, Paixão \& Herrera, 2009).

Emmons (2003) sistematiza las metas que se constituyen como claves para la integración de la persona en cuatro categorías: a) logros/ trabajo, que incluye la dedicación a la propia ocupación, la convicción de su carácter valioso y el gusto por el desafío que implica; b) relaciones/intimidad, o sea, la relación con los otros, confianza, altruismo, disposición a ayudar; c) religión/espiritualidad y auto-trascendencia, explicitada como la relación personal con Dios, la creencia en una vida después de la muerte y la actitud de contribuir a la fe de los otros; y d) generatividad, que entraña la disposición a contribuir socialmente, dejar un legado y trascender los intereses propios.

Desde la teoría de la selectividad emocional, se considera que la importancia relativa de las metas cambia en función del tiempo percibido (Fung \& Carstensen, 2006); por lo tanto, es de esperar que las personas de distinta edad se propongan un conjunto de metas de diferente tipo. Según esta teoría, la percepción del tiempo tiene un rol fundamental en la selección y prosecución de metas, en particular las de orden social y esto tiene consecuencias en los planos de la emoción, la estructura cognitiva y la motivación (Carstensen et al., 2011). En consonancia con esta idea, numerosos trabajos han explorado este aspecto. Entre los autores que más se han dedicado al tema se destaca Nurmi (1991), quien, desde su teoría de la auto-dirección del ciclo vital, se 
ocupa del rol que juegan las metas a través de las distintas etapas del desarrollo y en particular en las edades de transición. Distingue en las metas lo que llama tareas propias de la etapa de desarrollo — que pueden ser consideradas de orden transcultural-y las demandas normativas, transiciones institucionales y desafíos del contexto —que establecen diferencias socioculturales-.

Sin embargo, otros autores (Kauffman \& Husman, 2004; Nuttin \& Lens, 1985) subrayan que las metas vitales, más allá de tener como fuente las necesidades básicas o las demandas normativas propias de las etapas de desarrollo, pueden tener como referencia las diferentes orientaciones temporales. Así, por ejemplo, las metas relativas a logros escolares o diversiones denotarían la orientación al presente, las relativas al trabajo o grados académicos superiores remitirían a la orientación a futuro y las referidas al self podrían ser indicadores de referencia al pasado o de malestar personal (Salmela-Aro, 1992). En definitiva, a partir de lo referido, puede inferirse la relevancia de preguntarse por la posible relación entre las orientaciones temporales y las metas vitales y los cambios que se van dando en esta relación a través de las distintas etapas de la vida. Por otra parte, se han hallado diferencias en las metas que estarían ligadas al sexo. Así, por ejemplo, las mujeres tienen mayor inclinación a mencionar metas de contacto social que los varones (Díaz Morales \& Martínez, 2004; Greene \& DeBacker, 2004) y mayor preocupación por los logros académicos (Nurmi, 1991), en tanto que los varones mencionan con más frecuencia metas referidas a la búsqueda de placer y de posesiones materiales (Cross \& Markus, 1991; Lazarov \& Axman, 2016), aunque a este último respecto Nuttin (1980) observa que las mujeres pueden ser más reservadas en cuanto a manifestar estas metas.

\section{El presente estudio}

En línea con lo expuesto más arriba, nuestro objetivo principal es:

- Explorar las posibles relaciones entre la orientación temporal -OT- (focalización preferencial en el presente, pasado o futuro) y las metas vitales (el contenido motivacional). 
Planteamos como objetivos secundarios:

- Verificar en qué medida pueden mediar en esa relación la edad y el sexo.

- Explorar las posibles relaciones entre orientaciones temporales y nivel de planificación de metas. Se podría esperar que la OT al futuro esté positivamente relacionada con el nivel de planificación y, en cambio, ambas OT al presente (hedonista y fatalista) caractericen a sujetos menos inclinados a planificar.

- Explorar la posible relación entre la extensión de la OT y los estilos de OT.

- Verificar si hay diferencias por sexo y edad en la extensión de la OT.

\section{Método}

\section{Participantes}

Participaron 773 estudiantes argentinos de nivel medio (24\%), terciario (21\%) y universitario (55\%) - 60\% mujeres—, con una edad media de 24.71 ańos $(\mathrm{SD}=10.38)$ en un rango de 16 a 55 años, que cursan sus estudios (de grado o posgrado en el caso de los alumnos de mayor edad) en la ciudad de Buenos Aires y en el interior del país. A los fines del análisis, se categorizó la edad (Ver figura 1).

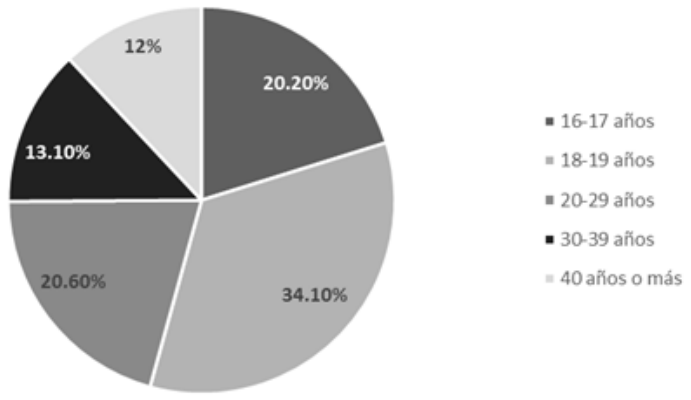

Figura 1. Porcentaje de participantes por categoría de edad 


\section{Medición}

Para evaluar la orientación temporal se aplicó el ZTPI (Inventario de Perspectiva Temporal, Zimbardo \& Boyd, 1999), en la versión en español de Díaz Morales (2006a), tres ítems del Cuestionario de Perspectiva Temporal Futura Trascendental (PTFT) (Boyd \& Zimbardo, 1997) y cinco elaborados ad hoc, integrados en un solo instrumento (Ver Apéndice 1).

El ZTPI es un cuestionario de 56 ítems con formato de escala Likert de cinco alternativas de respuesta ("completamente en desacuerdo" a "completamente de acuerdo"), compuesto por cinco sub-escalas que exploran cinco factores:

- Pasado negativo (PN), expresa una visión negativa y de rechazo hacia las experiencias del pasado.

- Pasado positivo (PP), evalúa la actitud cálida, sentimental y de nostalgia hacia el pasado.

- Presente hedonista (PH), muestra la medida en que la persona se orienta a buscar placer en el momento presente, con poca consideración de las consecuencias para el futuro.

- Presente fatalista (PF), refleja una actitud de indefensión, desesperanza y resignación frente a la vida y al futuro.

- Futuro (F), indica la búsqueda de objetivos y recompensas futuras, la capacidad de planificar y de posponer recompensas.

$\mathrm{Su}$ validez — convergente y discriminante - se informa en el trabajo seminal (Zimbardo \& Boyd, 1999). Díaz Morales (2006a), Corral-Verdugo, Fraijo-Sing y Pinheiro (2006), Albiñana Cruz (2013), Oyanadel, Buela-Casal y Pérez-Fortis (2014) validan versiones del ZTPI en español. Hay trabajos de adaptación a otros idiomas, para distintas poblaciones, entre los que se cuentan los de Milfont, Andrade, Belo y Pessoa (2008), Ortuño y Gamboa (2009), Apostolidis y Fieulaine (2004), D’Alessio, Guarino, De Pascalis y Zimbardo (2003), Oyanadel et al. (2014), que han mostrado la estructura factorial original y propiedades psicométricas semejantes, con algunas variaciones. En el estudio 
de Sircova et al. (2014), que abarca 24 países de los cinco continentes, se concluye que las orientaciones temporales que evalúa el ZTPI son invariantes a través de diversas tradiciones culturales y de muchas adaptaciones lingüísticas, por lo que el instrumento opera como un "estándar de oro" para la investigación de la orientación temporal (OT).

El cuestionario PTFT es un instrumento unidimensional (Boyd \& Zimbardo, 1997) que consta de diez ítems, con cinco opciones de respuesta (desde "completamente en desacuerdo" a "completamente de acuerdo"). Se seleccionaron los tres ítems que se relacionan palmariamente con la creencia en una dimensión del mundo y de la vida humana que trasciende las realidades estrictamente temporales (Ver Apéndice 1); en este sentido, un análisis factorial previo mostró que solo estos tres ítems evidenciaban cargas factoriales adecuadas. Por ello, en función de las características socioculturales de nuestra muestra, se elaboraron cinco enunciados ad hoc.

Para evaluar la validez de constructo del instrumento se efectuó un análisis factorial exploratorio con rotación Varimax, al que se ingresaron los 56 ítems del ZTPI y los 8 ítems de la versión adaptada del PTFT. Se examinó el valor propio de los componentes extraídos, las comunalidades, las saturaciones de cada ítem y el gráfico de sedimentación. Se obtuvo el índice de adecuación de la muestra de Keiser-Meyer-Olkin (KMO), que arrojó un coeficiente de .865 y la prueba de esfericidad de Barlett, que evidenció una significación $p<.0001$, resultados que indican que la matriz de datos es adecuada para realizar el análisis.

El análisis del gráfico de sedimentación muestra una estructura de seis factores para 60 ítems; tres de Futuro y uno de Pasado Positivo no cargan en factor alguno (Ver Apéndice 1); a excepción del ítem $\mathrm{N}^{\circ} 51$ (del ZTPI), estos ítems tampoco cargan en el estudio transcultural de Sircova et al. (2014). La varianza total explicada es de 38\%, mayor al coeficiente promedio de 35.05\% que informan Sircova et al. (2014) para el ZTPI. Por otra parte, en correspondencia con lo esperado, los ocho indicadores del PTFT cargan en este solo factor.

Para calcular la confiabilidad de cada factor, se aplicó el alpha de Cronbach. Respecto del ZTPI los coeficientes resultan más bajos 
(oscilan entre .60 y .87) en comparación con los de la versión original (entre .74 y .87). El PTFT, por su parte, evidencia muy buena confiabilidad (.87) y se trata del mismo coeficiente que Boyd y Zimbardo (1997, p. 41) informan para la escala original.

Con el fin de explorar las metas vitales se aplicó la primera parte (Ver Apéndice 2) del cuestionario de metas y temores de Nurmi (1989), en la cual se pide a los sujetos que hagan un listado de sus principales metas vitales (contenido), señalen la edad en que estiman o desean alcanzar cada meta (extensión), indiquen los factores que pueden incidir en su logro, el tipo de acciones realizadas, así como los planes para lograrlas (planificación). Para evaluar la confiabilidad en el análisis de contenido -categorización- de las metas, se calculó el porcentaje de acuerdo entre dos evaluadores independientes, que fue del $95 \%$.

Para la operacionalización de la variable metas vitales se elaboró una categorización de las metas expresadas por los participantes, resultando un listado de veinte categorías, que se agruparon luego en siete dimensiones cuya determinación se apoya en la propuesta de Nuttin (1980):

- Self: Agrupa las motivaciones concernientes a la propia persona, expresadas con el verbo ser — ser feliz, ser una buena persona-, preservarse de males, ya sea referidos a la salud psíquica, física- o a la situación social o económica, conseguir autonomía personal (vivir solo, poder mantenerse por sí mismo/a, no depender de otros).

- Logros: Motivaciones de logros en general, obtención de éxito en algún ámbito o, más específicamente, en el desempeño académico y laboral.

- Contacto: Motivaciones de orden social, relativas al plano de la intimidad — conseguir novio/a, tener amigos, compartir actividades, recibir afecto- $-\mathrm{y}$ a la posibilidad de hacer algo por los demás — contacto altruista—.

- Valores sensibles: Dimensión que reúne las respuestas referidas a metas de logro de posesiones materiales, bienestar económico, placer, diversiones, posibilidad de viajar y, en general, 
toda expresión que implica búsqueda de placer sensible.

- Familia: Dimensión que incluye exclusivamente las motivaciones de casarse, formar una familia, tener hijos.

- Autorrealización: Respuestas que se expresan como "Llegar a ser..." un buen profesional, una persona buena, equilibrada, una persona santa, alguien que logre los objetivos que se propuso.

- Valores de sentido: Motivaciones que implican la realización de una conducta o acción valiosa, ya sea en el ámbito moral, social, religioso, teórico y también el objetivo de contribuir a que esos valores tengan vigencia en su ámbito.

\section{Procedimiento}

Se usó un diseño transversal, no probabilístico, no experimental — no se manipularon variables_, de carácter intencional - los participantes se seleccionaron en función de los objetivos- y ecológico - ya que los datos se recogieron en el aula en una sola sesión de 45 minutos_- El completamiento de los cuestionarios fue de carácter voluntario y anónimo. Para el análisis cuantitativo del contenido de las metas vitales se procedió del siguiente modo: Se consignó para cada integrante de la muestra el tipo de objetivo y la cantidad de veces que aparece. Esta frecuencia se llevó a escala 10; luego, se obtuvo la media en cada dimensión respecto del total de metas enunciadas. Este total se usó como factor de covarianza en los distintos análisis.

Para estimar la edad probable de logro, se computó en cada meta una extensión que resulta de la diferencia entre la edad consignada y la edad cronológica. Después, se hizo un cálculo de la extensión media, dividiendo la sumatoria de cada una de las diferencias por la cantidad de metas consignada por el sujeto. La variable Planificación se evaluó a través de tres dimensiones: a) Cantidad de factores que menciona conocer como influyentes para el logro de cada una de las metas - conocimiento- b) Cantidad de acciones que consigna haber realizado para aproximarse a la meta — realización - y c) Cantidad de acciones que se propone realizar en el futuro - planes-. 


\section{Análisis de datos}

Para la variable edad se establecieron cinco categorías (Ver Tabla 1). Se emplearon como estadísticos: análisis de conglomerados, análisis de varianza one-way, análisis multivariado, análisis de covarianza y prueba de diferencia de proporciones.

\section{Resultados}

Dados los objetivos del trabajo, en primer lugar se realizó un análisis de conglomerados con las escalas que componen la orientación temporal, del que resultan dos conglomerados, que se conceptualizan como estilos de orientación temporal (EOT).

1. Estilo de Orientación temporal negativo-presentista $(\mathrm{N}=425)$ : Prevalecen el pasado negativo, el presente fatalista y el presente hedonista. Es un tipo de orientación que puede considerarse como menos adaptativa en relación con las metas personales.

2. Estilo de Orientación temporal positivo-proyectado $(\mathrm{N}=348)$ : Prevalecen el pasado positivo, el futuro (inmanente) y el futuro trascendente. Se considera que esta orientación es la más adaptativa.

Luego se efectúan los análisis pertinentes, cuyos resultados se presentan en cinco apartados: 1) relación entre estilos de orientación temporal y metas vitales, 2) diferencias demográficas en orientaciones temporales, 3) diferencias demográficas en metas vitales, 4) estilos de orientación temporal y nivel de planificación de las metas y 5) estilos de orientación temporal y extensión media.

\section{Relación entre estilos de orientación temporal y metas vitales}

Tomando como factor los EOT y como variables dependientes las categorías de metas (Ver Tabla 1), se realizó un análisis de varianza que verificó que el estilo negativo-presentista se relaciona con metas referidas al self, a los logros académicos y laborales y a los valores sensibles, tales 
como las posesiones materiales y las diversiones. En cuanto a las metas referidas al self, cabe establecer la distinción entre los sub-tipos que se agruparon bajo esa categoría, ya que solo tienen medias más altas para este conglomerado las metas de auto-preservación $[F(1,605)=8.3, p<.01]$ y de autonomía — vivir una vida independiente, vivir solo/a, no depender de nadie, entre las respuestas más frecuentes- $[F(1,605)=4.1, p<.05]$.

En cambio, el estilo de orientación positivo-proyectada identificó a las personas que mencionan metas referidas al contacto social (altruista e íntimo), a la auto-realización (profesional, de carácter y/o religiosa) y a los valores de sentido de la vida. Aquí, nuevamente, si se tienen en cuenta las categorías desagregadas, pesa sobre todo el contacto altruista - buscar o desear el bien de otros, ayudar, hacer algo por el bienestar de otras personas- $[F(1,605)=38.35, p<.001]$, más que el contacto íntimo - encontrar una pareja, tener amigos, estar con la familia-.

\section{Tabla 1}

Diferencias en metas vitales por estilos de orientación temporal

\begin{tabular}{|c|c|c|c|c|c|c|}
\hline \multirow[t]{2}{*}{ Metas } & \multicolumn{2}{|c|}{$\begin{array}{c}\text { EOT } \\
\text { Negativo- presentista }\end{array}$} & \multicolumn{2}{|c|}{$\begin{array}{c}\text { EOT } \\
\text { Positivo-proyectado }\end{array}$} & \multirow[b]{2}{*}{ F } & \multirow[b]{2}{*}{$p$} \\
\hline & $M$ & $D E$ & $M$ & $D E$ & & \\
\hline Self & 1.14 & 2.11 & .65 & 1.47 & 10.17 & .003 \\
\hline Logros & 5.11 & 3.11 & 4.14 & 3.21 & 13.93 & .001 \\
\hline Contacto social & .59 & 1.32 & 1.24 & 2.01 & 24.74 & .003 \\
\hline Auto-realización & .67 & 1.75 & 1.09 & 2.07 & 7.13 & .009 \\
\hline Valores de sentido & .10 & .57 & .75 & 1.55 & 51.30 & .001 \\
\hline
\end{tabular}

\section{Diferencias demográficas en orientaciones temporales}

Se hace un análisis de varianza tomando como factor las categorías de edades y como variables dependientes las escalas que integran la orientación temporal; se hallan diferencias por edad en presente hedonista, presente fatalista, futuro y futuro trascendente (Ver Tabla 2). 


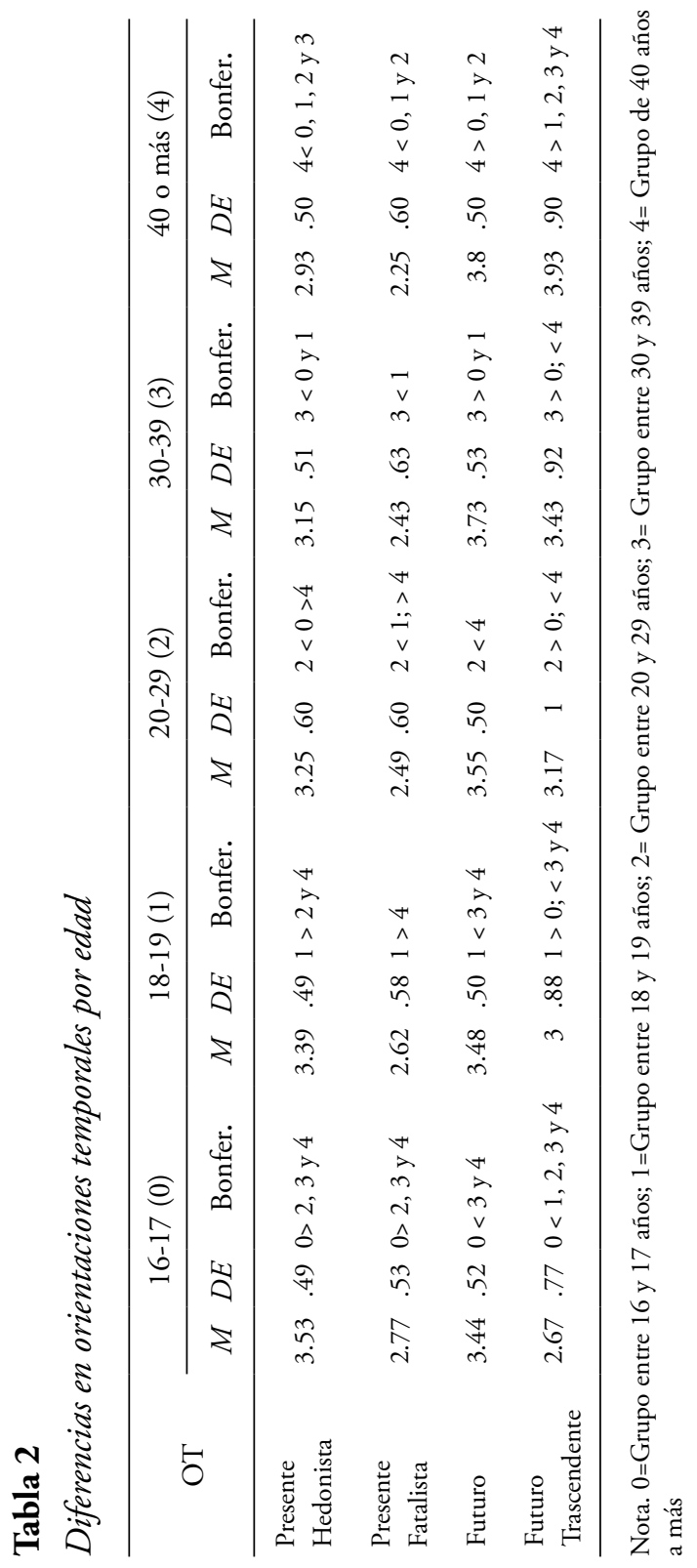


El grupo de adolescentes muestra los valores más altos en presente hedonista y fatalista y los más bajos en futuro y futuro trascendente, mientras que en el grupo de los mayores (desde los 30 ańos) sucede a la inversa. Ambos tipos de futuro suben con la edad y ambos tipos de presente descienden con la edad. En general, la orientación temporal más adaptativa (EOT positivo-proyectada) exhibe porcentajes más altos en los grupos de mayor edad y viceversa, como se verifica a partir de un análisis de contingencia (Ver Tabla 3).

\section{Tabla 3}

Diferencias en estilos de orientación temporal por edad

\begin{tabular}{lccc}
\hline Edad & EOT & EOT & $Z$ \\
\hline $16-17$ & Negativo- presentista & Positivo-proyectado & $12.07, \mathrm{p}<.0001$ \\
$18-19$ & $79.5 \%$ & $20.5 \%$ & $6.43, \mathrm{p}<.0001$ \\
$20-29$ & $63 \%$ & $37 \%$ & $0.79, \mathrm{~ns}$. \\
$30-39$ & $48 \%$ & $52 \%$ & $3.02, \mathrm{p}<.001$ \\
40 o más & $40 \%$ & $60 \%$ & $11.19, \mathrm{p}<.0001$ \\
\hline
\end{tabular}

Con respecto al sexo, las mujeres se ubican mayoritariamente en el grupo de EOT positivo-proyectada (EOT adaptativo); en cambio, los varones se concentran en el grupo de EOT negativo-presentista (menos adaptativo) (Ver Tabla 4).

\section{Tabla 4}

Diferencias en estilos de orientación temporal por sexo

\begin{tabular}{lccc}
\hline Sexo & $\begin{array}{c}\text { EOT } \\
\text { Negativo-presentista }\end{array}$ & $\begin{array}{c}\text { EOT } \\
\text { positivo-proyectado }\end{array}$ & $\mathrm{Z}$ \\
\hline Mujeres & $48 \%$ & $52 \%$ & $4.95, \mathrm{p}<.0001$ \\
Varones & $66 \%$ & $34 \%$ & \\
\hline
\end{tabular}


Un análisis de varianza one way muestra que las mujeres tienen medias más altas en pasado positivo $[F(1,771)=9.82, p<.002]$, en futuro $[F(1,771)=24.34, p<.001] \mathrm{y}$ en futuro trascendente $[F(1$, $771)=21.01, p<.001], \mathrm{y}$ la media más baja en pasado negativo $[F(1$, 771) $=5.68, p<.02]$.

\section{Diferencias demográficas en metas vitales}

No se registran diferencias significativas por edad en metas referidas al selfy a los valores sensibles. Por el contrario, en el resto de las categorías sí aparecen diferencias con significatividad estadística (Ver Tabla 5). Las metas de logro tienden a descender con la edad y no hay diferencias por sexo, pero la EOT sí influye: los participantes que pertenecen al grupo de orientación temporal negativo-presentista (no adaptativo) tienen medias más altas en metas de logro $[\mathrm{F}(600,4)=7.10, \mathrm{p}<.001]$. Las metas referidas al contacto social, la auto-realización y los valores de sentido se incrementan con la edad. La meta de formar una familia registra el nivel más alto en la franja etaria de 20 a 29 años, y el nivel más bajo para los participantes de más de 40 ańos. Respecto del factor edad, las metas de logro van disminuyendo a medida que aumenta la edad.

La única diferencia por sexo en metas vitales se verifica respecto de las metas de contacto social (altruista e íntimo), con nivel más alto para las mujeres $[F(1,604)=5.63, p<.02]$. Este resultado concuerda con el informado por Díaz Morales y Martínez (2004) en una muestra de adolescentes peruanos. Sin embargo, las diferencias anotadas hasta aquí presentan variaciones si se considera conjuntamente el peso de la orientación temporal, la edad y el sexo sobre las metas vitales. En efecto, un análisis de varianza multivariado - MANOVA - muestra que la diferencia a favor de las mujeres en contacto social aparece solo en el caso de que medie un estilo de Orientación Temporal (EOT) adaptativo $[F$ $(1,346)=20.77, p<.001]$. Los varones tienen nivel más bajo de metas de contacto social, sin diferencias por EOT. En general, la EOT influye en las mujeres, no en varones, salvo para valores de sentido $[F(1,256)$ $=32.15, p<0.001]$. La media de las metas de contacto social es más alta en el grupo de EOT adaptativo: en el caso de las mujeres a partir 
Orientación temporal y metas vitales en estudiantes argentinos / Difabio de Anglat et al.

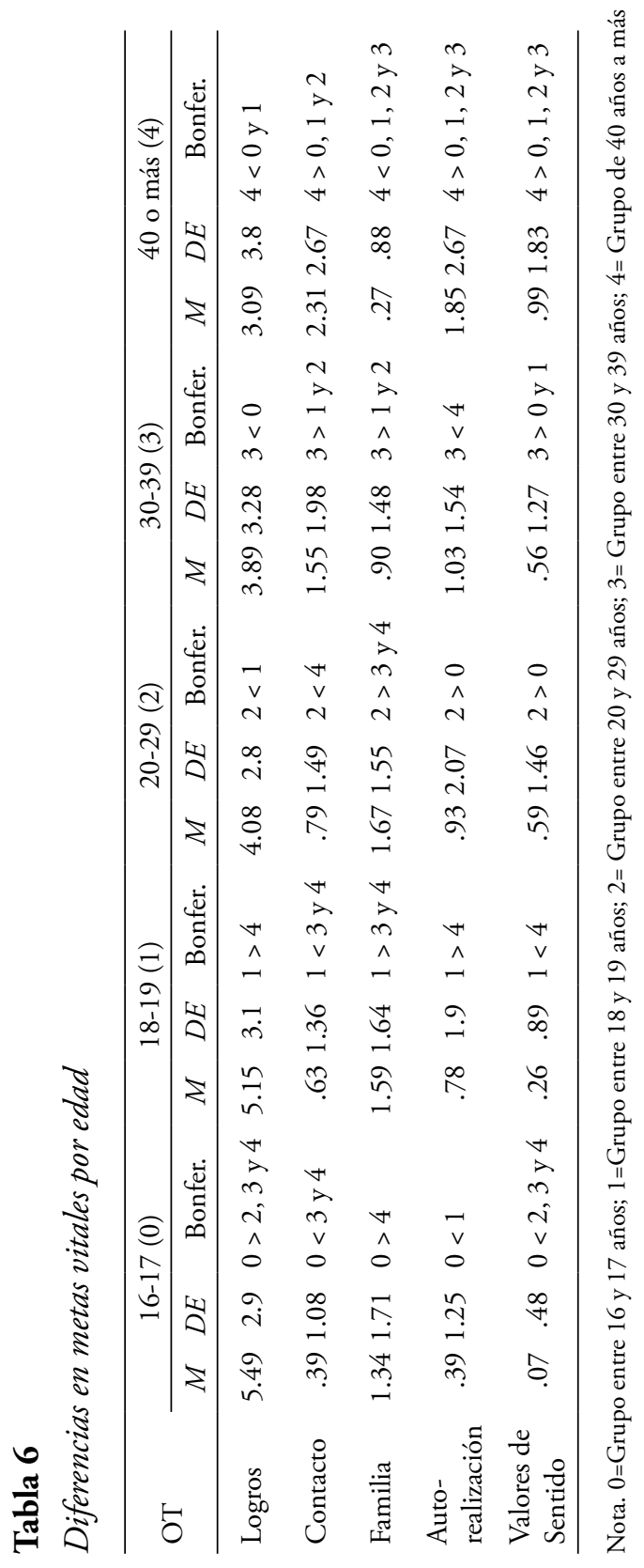


de los 20 ańos $[F(600,4)=7.32, p<.001]$ y en el caso de los varones a partir de los 30 años $[F(600,4)=3.95, p<.003]$. Los más jóvenes tienen nivel más bajo en todos los casos.

El nivel de metas de valores sensibles desciende para las mujeres de más de 40 solo en el caso del EOT positivo-proyectado. En las mujeres más jóvenes, el nivel de búsqueda de estos valores se mantiene alto, con independencia del EOT; en cambio, en los varones más jóvenes (17-18 años) sí se verifica el descenso de estas metas en relación con el EOT adaptativo. En el grupo de varones de más edad el nivel de valores sensibles es alto, con independencia de la OT. Las metas de auto-realización se acrecientan a partir de los 30 ańos $[F(600,4)=5.85, p<.001]$, pero solo en el grupo con EOT positivo-proyectado, tanto de varones como de mujeres. El nivel de valores de sentido va subiendo con la edad, a partir de los 20 años $[F(600$, $4)=2.60, p<.04]$, tanto para las mujeres como para los varones con EOT adaptativo. En resumen, el EOT tiene más peso sobre las metas vitales en las mujeres; además, esa influencia se incrementa con la edad.

\section{Estilos de orientación temporal y nivel de planificación de las metas}

Para verificar el rol de la OT en la planificación de metas, se hizo un análisis multivariado - MANOVA - tomando como factor los conglomerados de OT, covariando la cantidad de metas. Se verificó un efecto significativo [Traza de Pillai .093, $F(3,600) 20.48$, $p<.001$ ]: la orientación temporal más adaptativa — positivo-proyectada — influye positivamente sobre los tres componentes de la planificación (Ver Tabla 6). La varianza total explicada es del $40 \%$.

\section{Tabla 6}

Diferencias en planificación por Estilos de orientación temporal

\begin{tabular}{lccccccc}
\hline \multirow{2}{*}{ Planificación } & \multicolumn{2}{c}{ EOT Negativo- presentista } & EOT Positivo-proyectado & & \\
\cline { 2 - 6 } & $M$ & Error típ. & $M$ & Error típ. & $F$ & $p$ \\
\hline Conocimiento & 3.94 & .131 & 4.67 & .151 & 13.23 & .001 \\
Realización & 2.27 & .117 & 3.60 & .136 & 54.17 & .001 \\
Planes & 2.35 & .109 & 3.42 & .126 & 41.47 & .001 \\
\hline
\end{tabular}


Por otra parte, se verifica que el nivel de los tres componentes de la planificación es más alto en las mujeres que en los varones (Ver Tabla 7 ), con varianza explicada que va del $36 \%$ al $38 \%$.

\section{Tabla 7}

Diferencias en planificación por sexo

\begin{tabular}{lcccccc}
\hline \multirow{2}{*}{ Planificación } & \multicolumn{2}{c}{ Mujeres } & \multicolumn{2}{c}{ Varones } & & \\
\cline { 2 - 5 } & $M$ & Error típ. & $M$ & Error típ. & $F$ & $p$ \\
\hline Conocimiento & 4.47 & .132 & 3.95 & .154 & 6.40 & .02 \\
Realización & 3.02 & .123 & 2.60 & .143 & 5.02 & .03 \\
Planes & 3.05 & .113 & 2.47 & .131 & 11.03 & .002 \\
\hline
\end{tabular}

Respecto de la edad, se confirma que los participantes de menos edad (entre 17 y 20 ańos) tienen una media más baja en los aspectos de la realización de acciones y formulación de planes para el logro de metas $[F(3,592)=23.92, p<.01]$, aunque la varianza explicada más alta es del $10 \%$ para el caso de la capacidad de formular planes.

\section{Estilos de Orientación temporal y extensión media}

En el total de la muestra, se ratifica lo ya observado en trabajos previos respecto de la extensión temporal. En efecto, los varones alcanzan una media de 8.28 años, en tanto que las mujeres tienen una media de 6.5 años. Además, la extensión tiende a decrecer con la edad, aunque en nuestra muestra, a diferencia de lo hallado por otros autores (Thomae, 1981; Nuttin, 1985), no se verifican diferencias significativas.

No obstante, el EOT tiene un efecto significativo sobre la extensión de las metas, modificando los efectos simples de la edad sobre la extensión (Ver Figura 2): a partir de los 18 años los sujetos con un estilo de OT positivo-proyectado tienen una extensión temporal más alta que los sujetos con estilo negativo-presentista $[F(1,4)=6.37, p<.02]$, en los que se verifica un descenso a medida que sube la edad. 


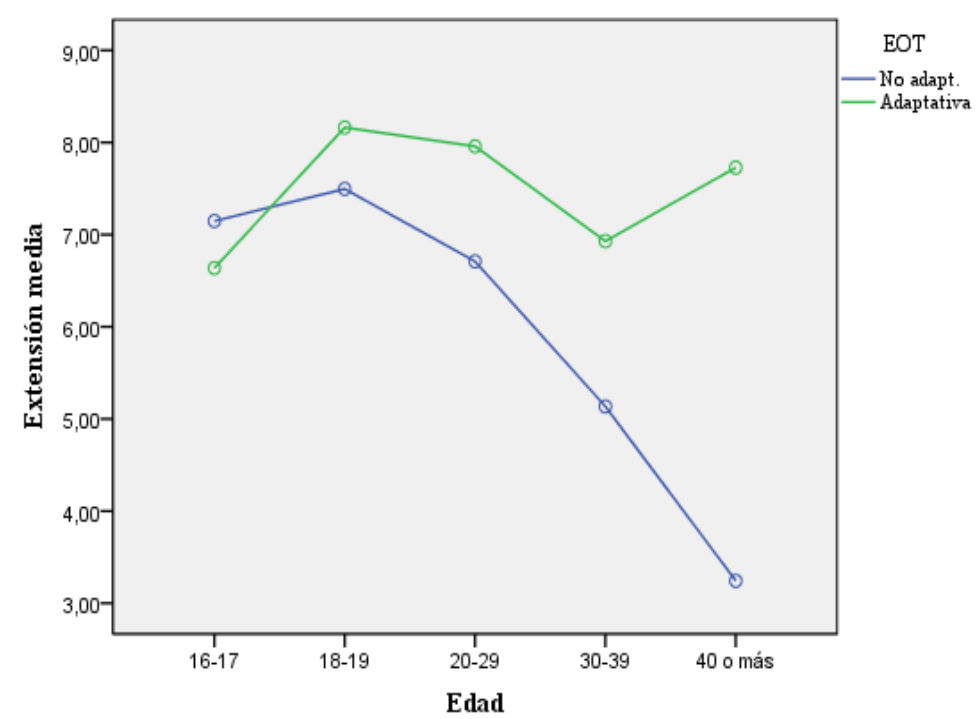

Figura 2. Extensión temporal de metas por edad según el estilo de orientación temporal

\section{Discusión}

La orientación temporal, como señaláramos, es una variable psicológica fundamental por su influencia dinámica sobre la motivación, la cognición y diversos aspectos de la conducta humana (Boniwell, 2009), a la vez que contribuye a otorgar orden, coherencia y significado a las experiencias (Zimbardo \& Boyd, 1999). Por otra parte, siguiendo a Nuttin (1980), la conducta se regula, en última instancia, por las metas que la persona se da a sí misma (se autoimpone), las que -más allá de responder a necesidades básicas o demandas normativas propias de las distintas etapas de la vida- pueden tener como referencia las diferentes orientaciones temporales (Kauffman \& Husman, 2004; Nuttin $\&$ Lens, 1985). De allí que el presente trabajo se orientara a explorar $1^{\circ}$ ) las posibles relaciones entre estas variables, $2^{\circ}$ ) las diferencias demográficas en orientaciones temporales, $3^{\circ}$ ) las diferencias demográficas en 
metas vitales $4^{\circ}$ ) la relación entre estilo de orientación temporal y nivel de planificación de metas $5^{\circ}$ ) la relación entre estilos de orientación temporal y extensión media. Respecto del primer objetivo, se obtiene una relación significativa entre el estilo de orientación temporal y la cualidad de las metas que los integrantes de nuestra muestra enumeran.

El estilo de orientación negativo-presentista se relaciona con metas referidas al self-en particular, las metas de autonomía y de autopreservación-, a los logros académicos y laborales y a los valores sensibles, tales como las posesiones materiales y las diversiones. Estas metas, por naturaleza, se ubican en un tiempo próximo y en el último caso refieren a la necesidad de placer. Estas observaciones concuerdan con los resultados hallados por Lazarov y Axman (2016) en una muestra de estudiantes universitarios suecos en la cual los sujetos menos orientados al futuro tienen puntajes más bajos en metas referidas al altruismo y a los valores de sentido.

La relación entre metas referidas al self y el estilo de orientación temporal menos adaptativo confirmaría lo observado por distintos autores (Salmela-Aro, 1992; Salmela-Aro \& Nurmi, 1997; SalmelaAro, Nurmi, Saisto \& Halmesmäki, 2001; Shulman \& Nurmi, 2010), respecto de que las metas centradas en el self, cuando pertenecen a logros existenciales definidos de modo abstracto, se asocian con bajos niveles de bienestar (emociones negativas, ansiedad, estrés). En nuestro trabajo, las respuestas enunciadas como "ser feliz" pertenecerían a esta categoría, ya que — como señala Emmons (2002) — si bien la felicidad es un objetivo primario, su logro depende de proyectos y actividades valiosas que no se intentan en primer lugar por ser fuente de felicidad.

La cuestión que se plantea es si el estilo de orientación temporal permite el surgimiento de estas metas o es a la inversa. La respuesta parece estar en una relación dialógica: Si la persona se ubica prevalentemente en un presente hedonista y fatalista, se comprende lógicamente que se centre en su propio yo, que busque gratificaciones inmediatas (diversión, placer). Pero ¿por qué los logros (académicos y laborales) se relacionan con esta orientación? Señala Lens (1998) que la motivación de logro prescinde de la dimensión temporal; en el caso de los inte- 
grantes de nuestra muestra, estas motivaciones parecen excluir el valor intrínseco, sobre todo respecto de lo académico, lo que se advierte en el modo de expresar dichas metas: "aprobar", "pasar de año", "recibirme", que son metas ordenadas a otras, pero en cuya enunciación no aparece -en nuestra muestra- su horizonte de sentido, ni, en particular, el valor del conocimiento, el aprendizaje como elemento de desarrollo personal. En otras palabras, los logros académicos y laborales son metas de motivación extrínseca, pero lo que es decisivo es la consideración del tipo de meta intrínseca al cual se ligan: ¿son instrumentales respecto de una meta con sentido de integralidad? ¿O están ligadas a metas de valor limitado? (Seginer \& Lens, 2015, Herrera, 2002; Lens, Paixão \& Herrera, 2009).

Sin duda, los resultados obtenidos en nuestro estudio parecen remitir a una visión pragmática del quehacer académico y allí cabría considerar en qué medida ciertos rasgos de la cultura actual influyen en la visión de los estudiantes, a la vez que preguntarse si otro condicionamiento puede ser un cierto estilo de evaluación por parte de los docentes.En cambio, el estilo de orientación positivo-proyectada identificaría a quienes mencionan metas referidas al contacto social (altruista e íntimo), a la auto-realización (profesional, de carácter y/o religiosa) y a los valores de sentido de la vida. Son metas congruentes con la proyección personal más allá de los límites del propio yo y del presente y son también metas intrínsecas, congruentes con la mayor extensión de la perspectiva temporal.

En estos sujetos, las metas de logro parecen estar suscitadas a partir de una motivación autónoma (Deci \& Rian, 2000; Lens, Paixao \& Herrera, 2009) Carver y Scheier (2000) afirman que la conducta motivada por la necesidad de auto-realización o autoactualización (Maslow, 1943) produce experiencias positivas que implican estados de plenitud, de satisfacción vital. Numerosos trabajos (Baumeister \& Vohs, 2002; Lent, 2004; Zika \& Chamberlain, 1992, entre otros) señalan que estas conductas están ligadas con la vivencia de valores, esto es, con la apreciación del contenido objetivo de valor de la meta en sí y para el sujeto.

Las motivaciones de contacto altruista son también propias de las personas que no se centran en el propio yo y esto implica la captación del 
sentido de la vida como integrada en un contexto más amplio que el de los intereses individuales e inmediatos. Malas y Guiot (2007) observan que las metas altruistas permiten superar el límite del horizonte temporal. Como señalan Fishbach, Ferguson y Kruglanski (2007), la meta es la representación cognitiva de un estado final deseado, pero puede ser también un estado ordenado a otro e, incluso, casi cualquier estado final puede ser entendido como medio de otro de orden superior, lo que sucede comúnmente cuando las metas configuran una estructura jerárquica. Sería esta jerarquía la que no siempre aparece, al menos explícitamente, en la enunciación de metas que los sujetos hacen, lo que, a nuestro juicio, puede minar el carácter motivador de las mismas, la fuerza para mover y sostener las conductas. Aunque es nuestra interpretación, una forma de verificarla es observar el tipo de conductas que suscita un enunciado-meta.

En cuanto a las diferencias demográficas en estilos de orientación temporal, se consideró en primer lugar la edad, hallándose que el perfil de los adolescentes de nuestra muestra registra valores más altos en presente hedonista y fatalista, así como valores más bajos en ambos futuros. Esto coincide parcialmente con lo hallado por otros autores. Por ejemplo, en una muestra chilena, Oyanadel et al. (2014) observaron valores más altos de presente hedonista en el grupo más joven, pero los valores superiores de presente fatalista correspondieron al grupo de mayor edad, en tanto que Díaz Morales (2006a), en una muestra espańola, halla mayor orientación a futuro en los grupos de más edad, a partir de los 30 ańos, interpretada como una mayor capacidad de planificación. Por otra parte, en una muestra con sujetos estadounidenses entre 10 y 30 años de edad (Steinberg, Graham, O’Brien et al. 2009) se halla que los adolescentes están menos orientados al futuro que los adultos.

La media de fatalismo en nuestra muestra, que disminuye con la edad, podría interpretarse en relación con características socioculturales propias de nuestro país, ya que estos adolescentes solo han conocido una etapa crítica de la vida social, caracterizada por la crisis de valores: corrupción, inseguridad, niveles crecientes de discordia sociopolítica. 
Fung y Carstensen (2006) señalan que la percepción del futuro como cerrado, limitado, incide en la "constelación de metas", la que se organiza en derredor de objetivos con recompensas inmediatas. En cambio, los integrantes de nuestra muestra mayores de 40 ańos tienen una experiencia histórica más extendida, que les permitiría afrontar el presente con una orientación temporal más adaptativa, signada por el futuro temporal y trascendente, que implican esperanza y un bagaje de valores con el que pueden vertebrar la identidad personal.

Las mujeres obtienen medias más altas en pasado positivo y en ambos futuros, así como un nivel más bajo de pasado negativo. Vale decir que habría en la mujer una mayor capacidad de resistencia en el presente frente al peso de las experiencias negativas, así como mayor habilidad para mirar hacia el futuro y una sensibilidad particular respecto del sentido trascendente de la vida. Las mujeres tienen también medias más altas en las metas referidas al contacto social altruista, mientras que en los varones sobresalen las metas ligadas con la búsqueda de valores sensibles, lo que coincide con los resultados hallados por otros autores (Cross \& Markus, 1991; Greene \& DeBacker, 2004).

El hallazgo más relevante se refiere a la relación entre el estilo de orientación temporal y la selección y planificación de metas vitales: la meta de contacto social —en particular el altruista — caracteriza a las mujeres - partir de los 20 años - cuando estas tienen un EOT positivo-proyectado (adaptativo). Esta orientación en los varones también se relaciona con un aumento del altruismo -a partir de los 30 ańos-. En las mujeres de más de 40 años, este estilo se relaciona con un descenso de los valores sensibles. Las metas de logro decrecen con la edad solo en el grupo de EOT adaptativo.

Al respecto, Wilding y Andrews (2006) observan que hay diferentes enfoques dentro de la motivación de logro —en particular, el referido a lo académico - y que el enfoque profundo se vincula con las metas altruistas, mientras que el enfoque superficial se asocia con la búsqueda de bienes materiales y de estatus. Si esto se aplica a los resultados que hemos obtenido, se puede decir que las medias más altas en metas de logro ubican a los integrantes de nuestra muestra en una 
orientación temporal presentista —en el "estrecho horizonte del presente" (Lewin, 1935) — porque se trata de metas que no tienen más proyección que el hecho de "pasar una etapa", sin vinculación con un proyecto de vida más integral.

El EOT adaptativo se relaciona también con el aumento de las metas de auto-realización y de valores de sentido, en ambos sexos. En general, el EOT pesa más en las mujeres que en los varones y esa influencia aumenta con la edad, lo que puede significar que la mujer vive el tiempo de un modo diverso al del varón, con mayor sensibilidad a la interacción con el contexto temporal, a los cambios y ritmos naturales.

Las diferencias en los componentes de la planificación —conocimiento, realización y planes - indican que las mujeres tienen medias más altas en el nivel de conocimiento de factores que inciden en el logro de las metas y en el nivel de realización y de planes, así como una extensión de perspectiva de futuro menor que los varones. Puesto que la planificación es un índice del realismo de las metas, estos dos resultados se articulan lógicamente, ya que, como señalan distintos autores (Díaz Morales, 2006b; Husman \& Lens, 1999), a medida que la extensión crece, suele desdibujarse el realismo de las metas. En cuanto a las diferencias por edad, los tres componentes suben con la edad, a partir de los 30 años, lo que resulta lógico como un efecto de maduración, de crecimiento en experiencia.

Desde los planteamientos de la teoría de la autodirección del ciclo vital de Nurmi (1991), estas diferencias se corresponden con el desarrollo propio del ciclo vital (Díaz Morales, 2006b). Sin embargo, estas relaciones también están mediadas por el EOT (que explica un porcentaje considerable de la varianza), lo que confirmaría la relación dialéctica entre esta y la naturaleza de las metas y, por lo tanto, la potencialidad que tendría el EOT para orientar la conducta a la consecución de metas vitales.

Se verificó que la media de extensión temporal es más alta en los varones y que en el total de la muestra tiende a disminuir con la edad, pero estas relaciones están mediadas por el EOT, lo que confirma el rol 
del contenido de las metas, puesto que los sujetos con un EOT adaptativo presentan, mayoritariamente, un perfil constituido por metas intrínsecas de valores de sentido y altruismo. La interpretación que generaliza los resultados, siguiendo a Nuttin (1980), es que el estilo de orientación temporal dependería de la naturaleza de las metas intentadas: Cuando estas trascienden el propio yo y los intereses inmediatos para centrarse en valores de sentido y en el interés por el bien de otros, se produciría una ubicación temporal más adaptativa, una proyección hacia el futuro, tanto temporal como trascendente, a la vez que la búsqueda consciente de la auto-realización en sentido integral, vale decir, la progresiva unificación de la persona en torno a un proyecto de vida.

En cambio, las metas centradas en el yo, en logros parciales o en valores sensibles, tendrían como efecto un estilo de orientación menos adaptativo, que llamamos negativo-presentista. Asumir metas cada vez más significativas, con mayor densidad de valor para la realización personal, sería un criterio de madurez, de adecuación a la etapa de la vida que se está transcurriendo. A este respecto, en nuestro país hay una tendencia creciente a exhibir conductas adolescentes más allá de los 25 años, como también es frecuente hallar adultos que se esfuerzan por asumir actitudes propias de sujetos más jóvenes. Se ha señalado (Laureiro-Martínez, Trujillo \& Unda, 2017) que a lo largo de la vida emergen patrones diferenciales de orientación temporal, pero dichos patrones pueden ser alterados si los sujetos adoptan una orientación temporal distinta de lo que se puede esperar por el momento del ciclo vital.

En el enfoque que asumimos, la perspectiva teórica del modelo de Zimbardo y Boyd (1999), resulta enriquecida por la consideración del contenido de valor de las metas. Puesto que la gestación de la orientación temporal y la estructuración jerárquica de metas vitales es un proceso de mutua realimentación, desde el punto de vista pedagógico se sigue la necesidad de favorecer en el proceso educativo la apertura a metas valiosas y la integración de las mismas en un proyecto de vida, que permita mirar al futuro con realismo y que así conduzca al logro de la auto-realización personal. La principal limitación del presente trabajo remite a las condiciones restrictivas en la conformación de la muestra, 
dado el menor número de participantes en los dos últimos estratos (entre 30-39 años y 40 años o más). De allí que, por las implicancias de las relaciones halladas, una línea de interés para la investigación futura es replicarlo en una muestra proporcional. Asimismo, en una muestra de tales características en nuestro país, sería relevante avanzar en la vinculación de los estilos de orientación temporal con constructos que la investigación psicoeducativa contemporánea muestra relacionados con aquellos: satisfacción vital, autorregulación del aprendizaje, logro académico, entre otros.

Por otra parte, nuestros resultados pueden operar como punto de partida para una experiencia de intervención, orientada a promover una orientación temporal más adaptativa, que denominamos positivoproyectada, a través de la selección y planificación de metas vitales más allá de los límites del propio yo y del presente.

\section{Referencias}

Albiñana Cruz, N. (2013). El rol de la orientación temporal en las actitudes hacia el ahorro. Revista de Investigación en Psicología Social, 1(1), 35-50.

Andre L, van Vianen AEM, Peetsma TTD \& Oort FJ (2018). Motivational power of future time perspective: Meta-analyses in education, work, and health. PLoS ONE 13(1). https://doi. org/ $10.1371 /$

Apostolidis, T. \& Fieulaine, N. (2004). Validation française de l'echelle de temporalité: The Zimbardo Time Perspective Inventory (ZTPI). Revue Européenne de Psychologie Appliquée, 54, 207-217. http://dx.doi.org/:10.1016/j.erap.2004.03.001

Aristóteles (1995). Física. Madrid: Gredos.

Baumeister, R. F. \& Vohs, K. D. (2002). The Pursuit of Meaningfulness in Life. En C. R. Snyder y S. J. Lopez (Eds.), Handbook of Positive Psychology (pp. 608-618). Oxford: Oxford University Press. 
Boniwell, I. \&Zimbardo, P. G. (2004). Balancing One’s Time Perspective in Pursuit of Optimal Functioning. En P. A. Linley y S. Joseph (Eds.), Positive psychology in practice. (pp. 165-178). Hoboken, NJ: Wiley \& Sons. https://doi.org/10.1002/9780470939338. ch10

Boniwell, I. (2009). Perspectives on Time. En C. R. Snyder y S. J. Lopez (Eds.), The Oxford Handbook of Positive Psychology (2a ed., pp. 295-302). Nueva York, NY: Oxford University Press. https://doi.org/10.1093/oxfordhb/9780195187243.013.0027

Boyd, J. N. \& Zimbardo, P. G. (1997). Constructing time after death: The transcendental future time perspective. Time and Society, 6(1), 35-54. http://dx.doi.org/10.1177/0961463X97006001002

Boyd, J. N. \& Zimbardo, P. G. (2005). Time Perspective, Health, and Risk Taking. En A. Strahman y J. Joireman (Eds.), Understanding behavior in the context of time: theory, research and applications (pp. 85-107). Mahwah, NJ: Erlabaum.

Carstensen L. L., Turan B., Scheibe S., Ram N., Ersner-Hershfield H., Samanez-Larkin G. R., et al. (2011). Emotional experience improves with age: evidence based on over 10 years of experience sampling. Psychol. Aging, 26, 21-33. 10.1037/a002128

Carver, C. S. \& Scheier, M. F. (2000). On the structure of behavioral self-regulation. En M. Boekaerts, P. R. Pintrich y M. Zeidner (Eds.), Handbook of Self-Regulation (pp. 42-80). San Diego, CA: Academic Press. https://doi.org/10.1016/ B978-012109890-2/50032-9

Chen Zhang, K. \& Yu, E. (2014). Quest for a good life: Spiritual values, life goals, and college students. Asia-Pacific Psychiatry, 6, 91-98. https://doi.org/10.1111/j.1758-5872.2012.00183.x

Corral-Verdugo, V., Fraijo-Sing, B. \& Pinheiro, J. Q. (2006). Sustainable behavior and time perspective: Present, past, and future orientations and their relationship with water conservation behavior. Interamerican Journal of Psychology, 40, 139-147.

Cross, S. \& Markus, H. (1991). Possible selves across the life span. Human Development, 34, 230-255. https://doi.org/10.1159/000277058 
D’Alessio, M., Guarino, A., De Pascalis, V. \& Zimbardo, P. G. (2003). Testing Zimbardo's Stanford Time Perspective Inventory (STPI) - Short Form. An Italian study. Time \& Society, 12(2/3), 333-347. https://doi.org/10.1177/0961463X030122010

Deci, E. L. \& Ryan, R. M. (2000). The "What" and "Why" of goal pursuits: Human needs and the self-determination of behavior. Psychological Inquiry, 11, 227-268. https://doi.org/10.1207/ S15327965PLI1104_01

Díaz Morales, J. (2006a). Estructura factorial y fiabilidad del Inventario de Perspectiva Temporal de Zimbardo. Psicothema, 18(3), 565-571.

Díaz Morales, J. (2006b). Perspectiva temporal futura y ciclo vital. Anales de Psicología, 22(1), 52-59. https://doi. org/10.1348/000709904X24726

Díaz Morales, J. F. \& Martínez, P. (2004). Metas y satisfacción vital en adolescentes espańoles y peruanos según sexo y nivel socioeconómico. Revista de Psicología, 22(1), 121-149.

Elliot, A. J., Sheldon, K. \& Church, M. (1997). Avoidance personal goals and subjective well-being. Personality and Social Psychology Bulletin, 23, 915-927. http://dx.doi. org/10.1177/0146167297239001

Emmons, R. A. (2003). Personal goals, life meaning, and virtue: Wellsprings of a positive life. En C. L. M. Keyes y J. Haidt (Eds.), Flourishing: Positive psychology and the life well-lived (pp. 105128). Washington, DC: American Psychological Association. https://doi.org/10.1037/10594-005

Fishbach, A., Ferguson, M. \& Kruglanski, A. (2007). The goal construct in social psychology. En Social psychology: Handbook of basic principles (2a ed., pp. 490-515). Nueva York, NY: Guilford Press. https://doi.org/10.1007/BF02333407

Fraisse, P. (1983). Le future dans les perspectives temporelles. International Journal of Psychology, 18, 489-495. https://doi. org/10.1080/00207598308247495

Fraisse, P. (1989). El tiempo vivido. Educación Física y Deportes, 53, 7-9. 
Fung, H. \& Carstensen, L. (2006). Goals change when life's fragility is primed: Lesson learned from older adults, the september 11 attacks and sars. Social Cognition, 24(3), 248-278. http://dx.doi. org/10.1521/soco.2006.24.3.248.

Greene, B. A. \& DeBacker, T. K. (2004). Gender and orientations toward the future: Links to motivation. Educational Psychology Review, 16(2), 91-120. https://doi.org/10.1023/ B:EDPR.0000026608.50611.b4

Herrera, D, (2002) Inserción Social en Adolescentes. Un estudio sociopsicológico. Persona, 5, 167-190. https://doi.org/10.26439/ persona2002.n005.875

Husman, J. \& Lens, W. (1999). The role of the future in student motivation. Educational Psychologist, 34(2), 113-125. http:// dx.doi.org/10.1207/s15326985ep3402_4.

Kauffman, D.F. \& Husman, J. (2004). Effects of time perspective on student motivation: Introduction to a special issue Educational Psychology Review, 16(1), 1-7. https://doi. org/10.1023/B:EDPR.0000012342.37854.58

Klinger, E. (1998). The search for meaning in evolutionary perspective and its clinical implications. En P. T. P. Wong y P. S. Fry (Eds.), Handbook of personal meaning: Theory, research, and application (pp. 27-50). Mahwah, NJ: Erlbaum.

Komar, E. (2003). El tiempo y la eternidad. Buenos Aires: Sabiduría Cristiana.

Laureiro-Martínez, D., Trujillo, C., Unda, J. (2017). Time Perspective and Age: A Review of Age Associated Differences. Frontiers in Psychology, 8(101), 1-5. https://doi.org/10.3389/ fpsyg.2017.00101

Lazarov, S. \& Axman, O. (2016). Consequences of Materialism and Temporal Orientation: Altruism, Environmentalism and Happiness (Dissertation). Recuperado de http://urn.kb.se/resolve?urn=urn :nbn:se:Inu:diva-53296 
Lens, W. (1998). El rol de la perspectiva de futuro en la motivación de los estudiantes. Persona, 1, 67-94. https://doi.org/10.26439/ persona1998.n001.692

Lens, W., Paixão, M.P. \& Herrera, D. (2009) Instrumental Motivation is Extrinsic Motivation: So What???. Psychologica, 50, 21-40. https://doi.org/10.14195/1647-8606_50_2

Lent, R. W. (2004). Toward a unifying theoretical and practical perspective on well-being and psychosocial adjustment. Journal of Counseling Psychology, 51(4), 482-509. http://dx.doi. org/10.1037/0022-0167.51.4.482

Lewin, K. (1935). A Dynamic Theory of Personality. New York: McGrawHill Book Co.

Malas, Z. \& Guiot, D. (2007). La perspective Future: Essai de clarification des construits et util possibles en comportement du consommateur. Gème Journées Normandes de Recherche sur la Consommation Société et consommations. Groupe ESC Rouen.

Maslow, A. (1943). A Theory of Human Motivation. Psychological Review, 50(4), 370-396. https://doi.org/10.1037/h0054346

Milfont, T. L., Andrade, P. R., Belo, R. P. \& Pesoa, V. S. (2008). Testing Zimbardo Time Perspective Inventory in a Brazilian sample. Interamerican Journal of Psychology, 42(1), 49-58.

Nurmi, J.-E. (1989). Planning, motivation, and evaluation in orientation to the future: A latent structure analysis. Scandinavian Journal of Psychology, 30(1), 64-71. http://dx.doi. org/10.1111/j.1467-9450.1989.tb01069.x.

Nurmi, J.-E. (1991). How do adolescents see their future? A review of the development of future orientation and planning. Developmental Review, 11(1), 1-59. https://doi.org/10.1111/j.1467-9450.1989. tb01069.x

Nuttin, J. (1980). Motivation et perspective d'avenir. Lovaina, Bélgica: Presses Universitaires de Louvain.

Nuttin, J. \& Lens, W. (1985). Future Time Perspective and Motivation: Theory and Research Method. Lovaina, Bélgica: Leuven University Press. 
Ortuño, V. \& Gamboa, V. (2009). Estrutura factorial do Zimbardo Time Perspective Inventory-ZTPI numa amostra de estudantes universitários portugueses. Avances en Psicología Latinoamericana, 27, 21-32.

Oyanadel, C., Buela-Casal, G. \& Pérez-Fortis, A. (2014). Propiedades Psicométricas del Inventario de Orientación Temporal de Zimbardo en una muestra chilena. Terapia Psicológica, 32(1), 47-55. http://dx.doi.org/10.4067/S0718-48082014000100005.

Salmela-Aro, K. (1992). Struggling with self. Personal projects of students seeking psychological counseling. Scandinavian Journal of Psychology, 33, 330-338. https://doi. org/10.1111/j.1467-9450.1992.tb00922.x

Salmela-Aro, K. \& Nurmi, J.-E. (1997). Goal contents, well-being and life context during the transition to university: A longitudinal study. International Journal of Behavioral Development, 20, 471491. https://doi.org/10.1080/016502597385234.

Salmela-Aro, K., Nurmi, J.-E., Saisto, T. \& Halmesmäki, E. (2001). Goal construction and depressive symptoms during transition to motherhood: Evidence from two longitudinal studies. Journal of Personality and Social Psychology, 81, 1144-1159. http://dx.doi. org/10.1037/0022-3514.81.6.1144.

Seginer, R. \& Lens, W. (2015). The Motivational Properties of Future Time Perspective Future Orientation: Different Approaches, Different Cultures. En M. Stolarski, N. Fieulaine y W. van Beek (eds), Time Perspective Theory; Review, Research and Application (pp. 287-304). Cham, Suiza: Springer. doi: https://doi. org/10.1007/978-3-319-07368-2_19

Shulman, S. \& Nurmi, J.-E. (2010). Dynamics of goal pursuit and personality make-up among emerging adults: Typology, change over time, and adaptation. En S. Shulman y J.-E. Nurmi (Eds.), The role of goals in navigating individual lives during emerging adulthood. New Directions for Child and Adolescent Development, 130, 57-70. https://doi.org/10.1002/cd.277 
Sircova, A., Van De Vijver, F., Osin, E., Milfont, T. L., Fieulaine, N., Kislali, A., Zimbardo, P. \& 54 members of the International Time Perspective Research Project (2014). A Global Look at Time: A 24-Country Study of the Equivalence of the Zimbardo Time Perspective Inventory. Sage Open, January-March, 1-12. http://dx.doi.org/10.1177/2158244013515686

Steinberg L., Graham S., O'Brien L., Woolard J., Cauffman E. \& Banich M. (2009). Age differences in future orientation and delay discounting, Child Development, 80(1), 28-44. https:// doi.org/10.1111/j.1467-8624.2008.01244.x

Thiébaut, E. (1998). La perspective temporelle, un concept à la recherche d'une définition opérationnelle, L'Année Psychologique, 98(1), 101-125. https://doi.org/10.7202/1036549ar

Thomae, H. (1981). Future time perspective and the problem of cognition-emotion interaction. En G. D’Ydewalle y W. Lens (Eds.), Cognition in human motivation and learning (pp. 261274). Hillsdale, NJ: LEA.

Vansteenkiste, M., Simons, J., Lens, W., Soenens, B., Matos, L. \& Lacante, M. (2004). Less is sometimes more: Goal content matters. Journal of Educational Psychology, 96(4), 755-764. http://dx.doi.org/10.1037/0022-0663.96.4.755

Webster, J. D. (2011). A new measure of time perspective: Initial psychometric findings for the Balanced Time Perspective Scale (BTPS). Canadian Journal of Behavioural Science/Revue Canadienne des Sciences du Comportement, 43(2), 111-118. http://dx.doi.org/10.1037/a0022801.

Wilding, J. \& Andrews, B. (2006). Life goals, approaches to study and performance in an undergraduate cohort. British Journal of Educational Psychology, 76, 171-182. https://doi. org/10.1348/000709904X24726

Wrosch, C. \& Scheier, M. (2003). Adaptive Self-Regulation of Unattainable Goals: Goal Disengagement, Goal Reengagement, and Subjective Well-Being. Personality and 
Social Psychology Bulletin, 29(12), 1494-1508. https://doi. org/10.1177/0146167203256921

Zika, S. \& Chamberlain, K. (1992). On the relation between meaning in life and psychological well-being. British Journal of Psychology, 83, 133-145. https://doi.org/10.1111/j.2044-8295.1992.tb02429.x Zimbardo, P. G. (2002). Time to take our time. Psychology Today, 35, 62. Zimbardo, P. G. \& Boyd, J. N. (1999). Putting time in perspective: A valid, reliable individual differences metric. Journal of Personality and Social Psychology, 77(6), 1271-1288. http://dx.doi. org/10.1037/0022-3514.77.6.1271.

Zimbardo, P. G., Keough, K. A. \& Boyd, J. N. (1997). Present time perspective as a predictor of risky driving. Personality and Individual Differences, 23(6), 1007-1023. http://dx.doi. org/10.1016/S0191-8869(97)00113-X.

Recibido: 27 de enero del 2017

Revisado: 28 de febrero del 2018 Aceptado: 16 de mayo del 2018 


\section{Apéndices}

\section{Apéndice 1: Cuestionario de perspectiva temporal}

Se destacan en letra itálica negrita los ítems del Inventario de Perspectiva Temporal Trascendente (Boyd \& Zimbardo, 1997) y en negrita, los ítems elaborados ad hoc. Por otra parte, se marcan solo en itálica los ítems que no cargan en factor alguno.

\section{Instrucciones}

A continuación, encontrarás una serie de afirmaciones. Señala tu grado de acuerdo o desacuerdo con el contenido de cada afirmación marcando solamente una de las opciones de respuesta posibles. Por ejemplo:

6. Tenemos solo esta vida para disfrutar y hay que tratar de pasarlo lo mejor que se pueda

Ten presente que una puntuación de " 1 " significa "completamente en desacuerdo"; " 2 ", "en desacuerdo"; " 3 ", "ni en acuerdo, ni en desacuerdo"; "4", "de acuerdo"; y "5", "completamente de acuerdo". No hay respuestas correctas o incorrectas. Contesta con sinceridad, de modo que se pueda conocer mejor tu forma de ser.

1. Pienso que reunirse con los amigos en una fiesta es uno de los placeres más importantes de la vida (ZTPI - PH1)

2. Las imágenes, sonidos y olores de la infancia me traen recuerdos maravillosos (ZTPI - PP2)

3. El destino determina mucho mi vida (ZTPI - PF3)

4. A menudo pienso en las cosas tan diferentes que pudiera haber hecho en mi vida (ZTPI - PN4)

5. Mis decisiones están muy influidas por las personas y las cosas que me rodean (ZTPI - PN5)

6. Tenemos solo esta vida para disfrutar y hay que tratar de pasarlo lo mejor que se pueda (elaborado ad hoc - FT57 reverso)

7. Creo que el día de una persona debería planificarse por la mańana (ZTPI - Fut6)

8. Me gusta pensar en el pasado (ZTPI - PP7)

9. Hago cosas impulsivamente (ZTPI - PH8) 
10. No me preocupa si las cosas no se hacen a tiempo (ZTPI - Fut9 reverso)

11. Cuando quiero conseguir algo, me fijo unas metas y considero los medios para poder alcanzarlas (ZTPI - Fut10)

\section{Cuando muera tendré que dar cuenta de las acciones que realicé durante mi vida (PTFT} - FT58)

13. Si los pongo en una balanza, tengo muchos más recuerdos buenos que malos (ZTPI - PP11)

14. Cuando escucho mi música favorita, pierdo la noción del tiempo (ZTPI - PH12)

15. Preparar el trabajo para el día siguiente y cumplir con los plazos se antepone a la diversión de hoy en la noche (ZTPI - Fut13)

16. Ya que las cosas serán lo que serán, realmente no me preocupa lo que pase (ZTPI - PF14)

17. Me divierten las historias sobre cómo eran las cosas en los «viejos tiempos» (ZTPI - PP15)

18. Cuandomefijounameta, tratodeverquéesloqueDiosquiereparami(elaboradoadhoc-FT59)

19. Las experiencias dolorosas del pasado permanecen en mi memoria (ZTPI - PN16)

20. De una vez por todas, voy a vivir mi vida tan plenamente como sea posible (ZTPI - PH17)

21. Me molesta mucho llegar tarde a mis citas o compromisos (ZTPI - Fut18)

22. Idealmente, viviría cada día como si fuese el último (ZTPI - PH19)

23. Los recuerdos felices de los buenos tiempos están muy presentes en mi mente (ZTPI - PP20)

24. Existen leyes divinas que los hombres deben respetar (PTFT - FT60)

25. Cumplo a tiempo con las obligaciones para con mis amigos y jefes (ZTPI - Fut21)

26. He tenido mi ración de abuso y rechazo en el pasado (ZTPI - PN22)

27. Tomo mis decisiones en el mismo momento en que actúo (ZTPI - PH23)

28. Afronto cada día como viene, sin intentar planificarlo (ZTPI - Fut24 reverso)

29. El pasado tiene demasiados momentos desagradables y prefiero no pensar en ellos (ZTPI - PP25 reverso)

30. Creo que debo planificar mi vida teniendo en cuentala vida eterna (elaborado adhoc-FT61)

31. Es importante poner excitación a mi vida (ZTPI - PH26)

32. He cometido errores en el pasado que ojalá pudieran deshacerse (ZTPI - PN27)

33. Es más importante divertirse con lo que uno está haciendo que conseguir hacer la tarea a tiempo (ZTPI - PH28)

34. Tengo nostalgia de mi infancia (ZTPI - PP29)

35. No tiene sentido que me preocupe por una vida más allá del tiempo en la tierra (elaborado ad hoc - FT62 reverso)

36. Antes de tomar una decisión, valoro los costes y beneficios (ZTPI - Fut30)

37. Tomar riesgos hace que mi vida no sea aburrida (ZTPI - PH31) 
Orientación temporal y metas vitales en estudiantes argentinos / Difabio de Anglat et al.

38. Es más importante para mí divertirme cada día de mi vida que estar pensando en el destino que me espera (ZTPI - PH32)

39. Pocas veces salen las cosas como yo quiero (ZTPI - PN33)

40. Es difícil olvidar imágenes desagradables de mi infancia (ZTPI - PN34)

41. Si tengo que pensar en metas, resultados y productos, dejo de disfrutar la realización de las actividades actuales (ZTPI - PF35)

\section{Estoy convencidola de que mi vida no acaba con la muerte del cuerpo (PTFT - FT63)}

43. Incluso cuando me divierto en el presente, retrocedo en el tiempo para compararme con momentos parecidos que viví en el pasado (ZTPI - PN36)

44. Uno no puede planificar el futuro porque las cosas cambian mucho (ZTPI - PF37)

45. El camino de mi vida está controlado por fuerzas en las que no puedo influir (ZTPI - PF38)

46. No tiene sentido preocuparme por el futuro ya que de todos modos no puedo hacer nada (ZTPI - PF39)

47. Termino mis proyectos a tiempo, avanzando de manera estable y continua (ZTPI - Fut40)

48. Tengo mucha confianza en que Dios me ayudará para concretar mi proyecto de vida (elaborado ad hoc-FT64)

49. Me encuentro "fuera de onda" cuando los miembros de mi familia hablan de cómo solían ser las cosas (ZTPI-PP41 reverso)

50. Me arriesgo para poner excitación en mi vida (ZTPI - PH42)

51. Hago listas de cosas para hacer (ZTPI - Fut43)

52. Con frecuencia sigo lo que me dice el corazón más que la cabeza (ZTPI - PH44)

53. Soy capaz de resistirme a las tentaciones cuando sé que hay trabajo que hacer (ZTPI - Fut45)

54. Me dejo llevar por la excitación del momento (ZTPI - PH46)

55. La vida de hoy es demasiado complicada, preferiría la vida más sencilla de antes (ZTPI - PF47)

56. Prefiero amigos que son espontáneos más que predecibles (ZTPI - PH48)

57. Me gustan los rituales y tradiciones familiares que se repiten regularmente (ZTPI - PP49)

58. Pienso en las cosas malas que me han ocurrido en el pasado (ZTPI - PN50)

59. Me mantengo trabajando en tareas poco interesantes, si estas me ayudan a salir adelante (ZTPI - Fut51)

60. Gastar lo que gano en placer hoy es mejor que ahorrarlo para la seguridad del mañana (ZTPI - PF52)

61. Con frecuencia la suerte compensa más que el duro trabajo (ZTPI - PF53)

62. Pienso en las cosas buenas que me he perdido en mi vida (ZTPI - PN54)

63. Me gusta ser apasionado/a en mis relaciones íntimas (ZTPI - PH55)

64. Siempre habrá tiempo para ponerme al día en mi trabajo (ZTPI - Fut56 reverso) 
Apéndice 2: Cuestionario de metas (Nurmi, 1989)

\begin{tabular}{ll}
\hline EDAD & SEXO \\
\hline
\end{tabular}

\section{Instrucciones}

Ahora piensa cuáles son tus objetivos o metas más importantes y anótalos en la primera columna en orden de importancia. Después, responde cada uno de los interrogantes que se plantean de acuerdo con estos criterios:

- En los ítems 2, 3 y 4, expláyate en tu respuesta.

- En los ítems 5 y 6 utiliza una puntuación de 1 a 5 según los siguientes criterios:

- En el ítem 5, una puntuación de 1 significa que "Depende totalmente de las circunstancias" y una puntuación de 5 significa que "depende totalmente de vos mismo/a".

- En el ítem 6, una puntuación de 1 significa que "no hay probabilidad de que se realice" y una puntuación de 5 significa que "hay muchas probabilidades de que se realice".

- Recuerda que no hay respuestas correctas o incorrectas sino personales. Contesta con sinceridad, de modo que se pueda conocer mejor tu forma de ser.

\begin{tabular}{|c|c|c|c|c|c|c|}
\hline $\begin{array}{l}\text { Enumera tus } \\
\text { principales } \\
\text { objetivos o } \\
\text { metas por } \\
\text { orden de } \\
\text { importancia }\end{array}$ & $\begin{array}{l}\text { 1. ¿Qué } \\
\text { edad crees } \\
\text { que tendrás } \\
\text { cuando se } \\
\text { realice? }\end{array}$ & $\begin{array}{l}\text { 2. ¿Cuáles son } \\
\text { los principales } \\
\text { factores } \\
\text { que pueden } \\
\text { influir en la } \\
\text { realización de } \\
\text { tu objetivo? }\end{array}$ & $\begin{array}{l}\text { 3. Si has hecho } \\
\text { alguna cosa } \\
\text { para realizar } \\
\text { tu objetivo, } \\
\text { ¿puedes } \\
\text { indicar cuál o } \\
\text { cuáles? }\end{array}$ & $\begin{array}{l}\text { 4. ¿¿Puedes } \\
\text { indicar de } \\
\text { qué forma } \\
\text { vas a realizar } \\
\text { tu objetivo } \\
\text { en lo } \\
\text { sucesivo? }\end{array}$ & $\begin{array}{l}\text { 5. ¿En qué } \\
\text { medida la } \\
\text { realización de } \\
\text { tu objetivo } \\
\text { depende de las } \\
\text { circunstancias o } \\
\text { de ti mismo/a? }\end{array}$ & $\begin{array}{l}\text { 6. ¿Cuál } \\
\text { es la } \\
\text { probabilidad } \\
\text { de que tu } \\
\text { objetivo se } \\
\text { realice? }\end{array}$ \\
\hline
\end{tabular}

